\title{
La proximidad geográfica en el contagio del fracaso empresarial en la pyme: Una aplicación empírica con el modelo probit espacial * $^{*}$
}

\author{
Christian C. Rodríguez Fuentes ${ }^{a}$, MARIluz MAté SÁnchez-VAL a , \\ FERNANDO A. LÓPEZ HERNÁNDEZ ${ }^{\text {a }}$
}

a Universidad Politécnica de Cartagena, Facultad de Ciencias de la Empresa, Calle Real, 3, 30201 Cartagena (Murcia), España. E-mail: chris.rodriguez.fuentes@gmail.com, mluz.mate@upct.es, Fernando.lopez@upct.es

\section{RESUMEN}

Este trabajo analiza el efecto de contagio en el fracaso empresarial como consecuencia de la proximidad geográfica entre empresas de reducido tamaño. Para ello, se desarrolla una aplicación empírica sobre una base de 2.710 pymes españolas localizadas en el municipio de Murcia. Con esta información, se estima un modelo de regresión espacial probit a partir del cual se contrasta la significatividad del efecto del contagio empresarial. Nuestros resultados indican que la probabilidad de fracaso de una pyme, no solo depende de las características específicas de cada empresa, sino que también influye la probabilidad de fracaso de empresas geográficamente próximas. Factores asociados a interacciones de carácter social y/o económico entre los agentes vinculados a las distintas empresas de una misma región estarían detrás de estos resultados.

Palabras clave: Fracaso empresarial, pyme, distancia geográfica, efectos espaciales.

\section{The Geographic Proximity in the Spillover Effects of Business Failure in SMes: Empirical Application with the Spatial Probit Model}

\begin{abstract}
This paper tests the role of spillover effects derived from the geographic proximity among reduced size firms in business failure. To get this purpose, we develop an empirical application on a sample of 2.710 Spanish Small, Medium size Enterprises (SMEs) located in the region of Murcia. With this information, we estimate a spatial probit regression model to contrast the significance of business spillover effects in business failure models. Our results show that the probability of business failure in SMEs depends not only on its own characteristics but also on the probability of failure of geographically close firms. Factors associated with social and/or economic interactions among the agents linked to the different firms in the same region would be behind these results.
\end{abstract}

Keywords: Business Failure, SMes, Geographic Proximity, Spatial Effects.

Clasificación JEL: C49, L80, R39

\footnotetext{
* Agradecemos sinceramente los comentarios y sugerencias constructivas de los revisores y el apoyo financiero de del Programa de Ayudas a Grupos de Excelencia de la Región de Murcia, Fundación Séneca (\#19884-GERM-15) y del proyecto ECO2015-651758-P
}

Artículo recibido en mayo de 2016 y aceptado en septiembre de 2016

Artículo disponible en versión electrónica en la página www.revista-eea.net, ref. ə-34308 


\section{INTRODUCCIÓN}

Las consecuencias económicas negativas que produce el fracaso empresarial, no solo sobre la empresa en cuestión sino sobre el área local en donde está situada, hacen que buena parte de las investigaciones en este ámbito estén orientadas a la formulación de modelos estadísticos que permiten determinar la probabilidad de fracaso antes de que ocurra. Estos modelos son utilizados por diferentes agentes económicos. Así, las instituciones aplican los modelos de fracaso con el fin de evitar conceder préstamos a empresas con altas probabilidades de fracaso; los inversores se apoyan en modelos de fracaso empresarial para reducir el riesgo que supone invertir en empresas en quiebra; las empresas realizan con mayor seguridad negocios con otras empresas (como clientes o proveedores) en base a los resultados derivados de estos modelos de fracaso y, los organismos reguladores también se apoyan en estos modelos predictivos para identificar la legalidad de la quiebra (Gepp y Kumar, 2012).

Los modelos aplicados determinan la probabilidad de fracaso empresarial utilizando distintas técnicas estadísticas. Así, existen una extendida serie de resultados univariantes centrados en el análisis de la propia serie de fracaso (Beaver, 1966). También, diversas técnicas multivariantes son utilizadas para predecir el fracaso empresarial considerando una combinación de variables internas de la empresa. Sirvan de ejemplo el modelo paramétrico Análisis Múltiple Discriminante (MDA) propuesto por Altman (1968) y que posteriormente fue mejorado por Deakin (1972) y Edmister (1972). En esta categoría paramétrica además, se encuentra el modelo logit propuesto por Ohlson (1980) que ha tenido una importante repercusión en este campo. Frente a estos modelos paramétricos se encuentran los no paramétricos de entre los que destacan el modelo de redes neuronales artificiales de McCulloch y Pitts (1943) y el de árboles de decisión de Rokach y Maimon (2008). Para una revisión detallada de estos estudios se puede consultar el trabajo de Rodríguez et al. (2015) donde además se analiza la bondad de predicción de las distintas técnicas aplicadas, concluyendo que cualquiera de estas técnicas basadas en información interna de la empresa reporta buenos resultados de predicción.

Estudios anteriores han tenido como objetivo, además de evaluar la probabilidad de fracaso empresarial con carácter predictivo, identificar los factores que influyen en la probabilidad de dicho fracaso. Así, encontramos estudios que analizan el efecto de la edad (Cinca et al., 2005), el tamaño (Turetsky y McEwen, 2001) o el sector de la empresa (Quintana et al., 2012). En este contexto, recientemente se han desarrollado estudios que analizan el efecto del contagio en el fracaso empresarial (Kolay et al., 2015; Addoum et al., 2014; Le, 2012; Hertzel et al., 2012; Hertzel et al., 2008; Lang y Stulz, 1992). Como contagio nos referimos al hecho que una empresa superviviente o sana vea afectada su situación de fracaso debido a la situación de bancarrota de otra empresa (Le, 
2012). Las evidencias empíricas obtenidas en estos artículos han determinado que la proximidad geográfica entre empresas tiene un papel significativo en el contagio del fracaso empresarial aunque el sentido de dicho efecto debe ser examinado más detenidamente.

Nuestro trabajo se centra en profundizar en este factor. Así, el objetivo de este estudio es analizar el papel de la distancia geográfica entre empresas en el contagio del fracaso empresarial en el caso de la pequeña y mediana empresa (pyme en adelante). Este estudio se centra en empresas de reducida dimensión debido su la alta dependencia con las características de su entorno y, por tanto, es de esperar un efecto de contagio más intenso (Maté et al., 2013). Para conseguir nuestro objetivo, se desarrolla una aplicación empírica basada en una base de pymes españolas del sector servicios localizados en el municipio de Murcia durante el periodo 2011-2013 extraídas de la base de datos SABI (Sistema de Análisis de Balances Ibéricos). Nuestro estudio se basa en el sector servicios debido a la importancia que tiene en el tejido empresarial del municipio, que genera más del 65\% del Valor Añadido Bruto (VAB) en 2013, según datos de la Contabilidad Regional de España, del Instituto Nacional de Estadística (INE). También el municipio de Murcia es una región propicia para el desarrollo de nuestro estudio debido a varios factores: (i) el tejido productivo está constituido por un porcentaje muy elevado de empresa de pymes, (ii) La crisis ha tenido una repercusión muy importante en la situación de las empresas de la Región de Murcia. Por tanto, cabe esperar que los efectos de fracaso sean más intensos que en otras regiones. Finalmente, el intervalo temporal considerado, 2011-2013, es un periodo de crisis financiera por lo que cabe esperar que los efectos de contagio sean aún más intensos. A partir de esta información, se realiza la estimación de un modelo de regresión probit espacial (Wilhelm y Matos, 2013). Los resultados obtenidos corroboran la existencia de un efecto de contagio significativo y positivo en el fracaso empresarial que puede ser explicado por pautas de comportamiento local relacionadas con procesos de interacción entre los agentes vinculados a las distintas empresas (Pirinsky y Wang, 2010).

Este trabajo se estructura como sigue. En la segunda sección se revisa la literatura previa para determinar los factores del contagio asociados con la proximidad geográfica. En la tercera sección, se describe la muestra, las variables que intervienen en este estudio y la metodología empleada. En la sección cuarta, se describen los resultados obtenidos del análisis espacial. El trabajo concluye exponiendo las principales conclusiones.

\section{FACTORES EXPLICATIVOS DEL CONTAGIO DE FRACASO EMPRESARIAL RELACIONADOS CON LA PROXIMIDAD GEOGRÁFICA}

La literatura de fracaso empresarial considera fundamentalmente dos vías de 
propagación de contagio entre empresas. En primer lugar, el fracaso empresarial puede propagarse a través de la red comercial, donde el contagio se extiende a través de la cadena de suministros. En este caso, los efectos de dicha propagación pueden ser positivos o negativos. De este modo, una empresa con problemas financieros, llegando a una situación de fracaso empresarial puede ejercer efectos económicos negativos sobre las empresas con las que mantiene lazos comerciales (Battiston et al., 2007; Hertzel et al., 2008; Kolay et al., 2015). Sirva como ejemplo el acceso al crédito comercial. La situación inestable, desde un punto de vista financiero, de una empresa producirá una disminución en el acceso al crédito de las empresas con las que mantiene relaciones comerciales dando lugar a tensiones financieras en dichas empresas. Por el contrario, una situación de fracaso empresarial en una empresa también podría beneficiar a las empresas de la competencia, las cuales podrán incrementar sus beneficios absorbiendo la cuota de mercado correspondiente a la empresa en situación de fracaso y ejerciendo presión sobre los precios a los clientes y proveedores que deja la empresa en bancarrota (Lang y Stulz, 1992; Kolay et al., 2015). Así, en el ámbito financiero, Hertzel et al. (2012) determinaron que si la bancarrota tiene lugar entre empresas de la competencia, los proveedores de capital reaccionarán al contagio imponiendo mayores costes crediticios y términos contractuales más estrictos.

En segundo lugar, la literatura de fracaso empresarial también considera como vía de propagación del fracaso empresarial la proximidad geográfica entre empresas (Addoum et al., 2014). De este modo, la situación financiera de una empresa afecta a la situación financiera de las empresas de su entorno más cercano (Granovetter, 1985). Los argumentos explicativos de este efecto de contagio empresarial entre empresas físicamente próximas son diversos. Algunos trabajos señalan el papel del administrador que forma parte de varios consejos de dirección de empresas locales y normalmente del mismo sector. Este cargo le permite disponer de una mayor información en el proceso de toma de decisiones que repercuten en el éxito o fracaso de la empresa. Su desempeño está condicionado por su propia experiencia en la gestión de empresas que pueden ser empresas sanas o empresas en situación de fracaso. Como resultado, cabe esperar, que se genere una cierta interacción entre los agentes que forman parte de los distintos consejos de dirección pudiendo tener consecuencias positivas sobre la empresa sana (Le, 2012). Así, si un administrador forma parte de los consejos de dirección de una empresa sana y de otra fracasada habrá una mayor transmisión entre prácticas de gestión con una información más fluida (Haunschild y Beckman, 1998). Este intercambio de información, así como la experiencia derivada de estas prácticas de gestión en lo que se refiere al resultado de la propia empresa, facilitará la elaboración de nuevas estrategias. De acuerdo con este elemento, la reacción de una empresa sana ante el fracaso de otra empresa dependerá principalmente de la comunicación, favorecida por este factor, pero si además la empresa sana y la que presenta situación de fracaso pertenecen a un mismo sector y están geográfica- 
mente próximas, la transmisión de la información será más rápida y eficaz debida que habrá más interconexión entre los agentes que forman parte de los consejos de dirección (Le, 2012). Esta interrelación favorecerá el diseño de nuevas políticas. De este modo, una empresa sana cuyos administradores tienen conocimiento de la situación de fracaso de una empresa próxima a ella y que trabaja en el mismo sector tenderá a adoptar una serie de medidas conservadoras dirigidas a incrementar los niveles de tesorería y reducir los de endeudamiento e inversión. Estas políticas se deben a la creencia de un riesgo futuro de la empresa así como la estrecha relación entre la experiencia del administrador y sus decisiones, de manera que las políticas empresariales (como endeudamiento, tesorería e inversión) serán más conservadoras si el administrador ha pasado por una experiencia difícil en otro consejo de administración (Dittmar y Duchin, 2014). Por el contrario, también afectará a la gestión de la empresa el hecho que exista una mala práctica financiera entre empresas vecinas, ya que como aseguran Parsons et al. (2014) "las empresas tienden a adoptar un comportamiento similar al que tienen las empresas próximas entre sî". En este caso, la proximidad geográfica entre empresas ejercerá un efecto positivo en el contagio del fracaso empresarial. Es decir, empresas en situación de fracaso tenderán a contagiar esta situación hacia las empresas localizadas próximas a ellas.

En relación al concepto de contagio en el fracaso empresarial asociado a la distancia geográfica surge también la cuestión de la localización de la empresa, ya que, aquellas empresas de un mismo sector que se localizan próximas a otras empresas constituyendo un clúster podrán beneficiarse, entre otras cosas, de mayor información (Audretsch y Feldman, 1996). Por tanto, en este caso, también se reducirá la probabilidad de fracaso. Finalmente, también se debe tener en cuenta el impacto de los efectos de contagio en el sistema financiero. En este caso, la literatura se orienta principalmente a estudiar la respuesta de los bancos ante un fracaso empresarial a nivel industrial (Hertzel y Officer, 2012) y no de localización geográfica. No obstante, si se consideran los efectos de las condiciones de los préstamos y la distancia geográfica entre empresas, el banco y los otros bancos en la vecindad (Degryse y Ongena, 2005), encontramos que existe discriminación espacial de los costes en los préstamos bancarios. Desde esta perspectiva, los bancos frente a un caso de fracaso empresarial tienen la suficiente discrecionalidad para restringir la concesión de crédito y subir los tipos de interés (Gatti et al., 2005). Esta decisión tiene consecuencias sobre el resto de empresas localizadas en el mismo entorno.

\section{APLICACIÓN EMPÍRICA}

\subsection{Muestra}

Para el desarrollo de este trabajo se utilizó la base de datos SABI. Esta base de datos contiene información económico-financiera de más de 200.000 empre- 
sas españolas (el 95\% de todas las empresas españolas con facturación superior a los 360.000 euros y obligadas, por tanto, a presentar sus cuentas en los registros mercantiles). A partir de este universo, se seleccionaron aquellas empresas cuya actividad principal está asociada al sector servicios (excepto actividades financieras) localizadas en el municipio de Murcia durante el periodo 20112013.

La información suministrada por el Centro Regional de Estadística de Murcia $^{1}$ (CREM) indica que la crisis económica durante el periodo analizado ha afectado de forma más negativa a la Comunidad Autónoma de la Región de Murcia (CARM) que al conjunto nacional. Así, mientras la economía de la CARM retrocedió un 1,7\% en media anual durante el período 2008-2013, en España dicha caída fue del 1,1\%. Respecto a la composición sectorial, en Murcia el Sector Servicios seguido por el Industrial, son los que más contribuyen al Producto Interior Bruto (PIB). Estos dos sectores representan aproximadamente el 83\% del PIB (datos de 2012) siendo el Sector Servicios el que aporta más de dos tercios del total. Atendiendo a estas cuestiones, Murcia es una región propicia para el desarrollo de este trabajo, tanto por el peso del Sector Servicios como por la alta repercusión de la crisis financiera en las empresas de la CARM. La elección del municipio de Murcia dentro de la CARM permite analizar una unidad geográfica más compacta que el total regional representando más del 60\% de la actividad económica regional.

Seleccionadas las empresas de reducido tamaño localizadas en el municipio de Murcia, fueron eliminadas aquellas que presentaban incoherencias en sus cifras contables (así por ejemplo, las empresas cuyas cifras de activos no coincidían con la de sus pasivos fueron eliminadas). Además, también se descartaron aquellas empresas para las que no se disponía información en algunas de las variables examinadas durante tres años consecutivos. De este modo, la muestra final consta de 2.710 pymes del Sector Servicios con información disponible para el periodo 2011-2013.

\subsection{Variables}

\subsubsection{Variable endógena: El fracaso empresarial}

Estudios previos en este contexto han utilizado distintas definiciones de fracaso empresarial. Dos líneas son las que han dominado la literatura: Las que basan la definición en criterios legales y las que utilizan criterios económicos. Con respecto a las definiciones basadas en criterios legales no existe un consenso unánimemente aceptado. Algunos trabajos consideran en situación de fracaso aquellas empresas que se encuentran inmersas en procedimientos de insolvencia (Smaranda, 2014). Otros estudios consideran como empresas fraca-

\footnotetext{
${ }^{1}$ CREM: http://econet.carm.es/web/crem
} 
sadas aquellas que han presentado un expediente concursal según "ley concursal 22/2003" (Pozuelo et al., 2013). También se definen como empresas fracasadas aquellas que tengan una declaración formal de quiebra conforme a la legislación de cada país (García-Gallego y Mures-Quintana, 2012; Pervan et al., 2011; Carter y Van Auken, 2006) o que hayan sido declaradas en bancarrota por el índice "The Wall Street Journal” (Grepp et al., 2009). Algunos autores condicionaron el fracaso empresarial a una situación de moratoria de pagos a petición legal (Ooghe y Balcaen, 2007) y otros por el hecho de tener la situación legal “disuelta" (Puagwatana y Guanawardana, 2005). Estas definiciones presentan sus limitaciones al no existir una diferencia clara, en primer lugar entre empresa fracasada y empresa no declarada aún en quiebra, pero en proceso de serlo, y en segundo lugar, por los criterios adoptados en las normas según el tiempo ${ }^{2}$ o país para una misma situación de empresa (Tascon y Castaño, 2012). Además, Campillo et al. (2013) argumentan que las definiciones basadas en criterios legales generan una reducción considerable del tamaño de la muestra. Finalmente, Balcaen y Ooghe (2006) también argumentan que una empresa puede estar declarada legalmente en quiebra, y no mostrar signos reales de fracaso, lo que contaminaría la muestra para la estimación del modelo de predicción, por ejemplo, cuando una empresa se declara en quiebra solo para deshacerse de sus deudas o sufre un desastre natural.

Aparte de estas definiciones basadas en criterios legales otros trabajos utilizan criterios económicos para definir el fracaso empresarial, aunque tampoco existe una definición consensuada. La ventaja de estas definiciones es su carácter objetivo ya que están basadas en la información suministrada por los estados contables de las empresas y, por tanto, no existe el sesgo derivado de la propia definición legal de fracaso que encontramos siguiendo la definición legal anterior. Desde esta perspectiva económica, los criterios utilizados para considerar a una empresa como empresa fracasada han sido dispares. De este modo, varios estudios definen como fracaso empresarial al estado en el que se encuentran aquellas empresas que obtienen resultados del ejercicio negativos durante una serie de años consecutivos. Respecto al número de años, hay estudios que han usado la información correspondiente a los dos últimos años (Xu et al., 2014; Mateos y López, 2012; Li y Xiong, 2012; Mateos-Ronco y López, 2011; Li y Sum, 2011) y otros trabajos que han considerado resultados negativos los últimos tres años (Youn y Gu, 2010). También encontramos estudios que basan la definición de fracaso empresarial en el concepto de quiebra técnica. En este caso, la empresa se define como fracasada si el valor de sus deudas supera al de

\footnotetext{
${ }^{2}$ Tascon y Castaño (2012) exponen que "De acuerdo con la normativa actual en España, una empresa puede solicitar voluntariamente la declaración de concurso de acreedores para beneficiarse de las ventajas que la normativa le ofrece para salir de una situación transitoria de dificultades, o bien, para evitar las consecuencias negativas de no haberlo hecho, si posteriormente llega a quebrar y se la declara en quiebra culpable”.
} 
sus inversiones o lo que en términos contables equivale a un patrimonio neto negativo (Rubio, 2008).

En base a esta literatura, la definición de fracaso empresarial que utilizamos en este trabajo está basada en criterios económicos y no legales. En particular, consideramos como empresa fracasada aquella empresa con patrimonio neto negativo al menos durante tres años consecutivos tomando como referencia el periodo analizado 2011-2013. Empresas sanas son aquellas que no cumplen esta condición. Esta definición es objeto de nuestro interés dada su sencillez para determinar objetivamente la situación de bancarrota de las empresas que aunque no estén declaradas legalmente, si están reconocidas contablemente. Por tanto, su utilización ayuda a evitar los problemas asociados a la definición legal de fracaso, como, por ejemplo: al no existir una diferencia clara, en primer lugar entre empresa fracasada y empresa no declarada aún en quiebra, pero en proceso de serlo, y en segundo lugar, por los criterios adoptados en las normas según el tiempo o país para una misma situación de empresa (Fernández y Gutiérrez, 2012).

\subsubsection{Variables explicativas del modelo}

Las variables explicativas seleccionadas para el estudio son de tipo financiero que permiten tener en cuenta la situación interna de la empresa. En particular, se han seleccionado ratios financieros que evalúan la posición de liquidez (ratio de liquidez), el nivel de endeudamiento (ratio de endeudamiento) y la rentabilidad (ratio de rentabilidad económica) de la empresa. Con el fin de disminuir los posibles problemas de endogeneidad derivados del hecho de que existe cierta interrelación entre la variable representativa del fracaso empresarial y los valores de los ratios financieros, se amplió la información muestral para los tres ratios para el año 2010. Estos valores se consideraron como variables instrumentales en nuestro modelo sustituyendo a los valores iniciales de los ratios financieros.

Además de estos tres ratios financieros, se han utilizado otras tres variables de control: la edad de la empresa, el subsector de actividad y el tamaño empresarial. Con respecto a la edad de la empresa estudios previos presentan evidencias de que las empresas jóvenes tienen mayores dificultades financieras que las maduras, y por tanto, mayor riesgo de fracaso (Misas, 2008; Shumway, 2001). El subsector de actividad económica también debe tener impacto en la probabilidad de fracaso. Por último, el tamaño empresarial también tiene una importante influencia en la determinación del fracaso empresarial. Así, varios estudios concluyen sobre una relación negativa entre el tamaño y la probabilidad de fracaso (Calvo-Flores, 2007; Back, 2005; Watson y Everett, 1996). La Tabla 1 muestra una descripción detallada de las variables utilizadas en este trabajo. 
Tabla 1

Descripción de las variables

\begin{tabular}{ll}
\hline Fracaso Empresarial & $\begin{array}{l}\text { Variable es igual } 1 \text { si la empresa ha obtenido resultados negativos en los tres } \\
\text { últimos años del estudio (de } 2011 \text { a 2013) y cero en otro caso. }\end{array}$ \\
\hline Edad de la empresa & Periodo que transcurre desde su constitución. En transformación logarítmica. \\
Tamaño de la empresa & $\begin{array}{l}\text { Logaritmo del Total de Activos de la empresa. } \\
\text { Subsector se actividad siguiendo la Clasificación Nacional de Actividades }\end{array}$ \\
Subsector Servicios & $\begin{array}{l}\text { Económicas (CNAE 2009): G-Comercio, H- Transporte, I-Hostelería, J- } \\
\text { Información, L-Inmobiliarias, M-Científicas y técnicas, N-Administración, P- }\end{array}$ \\
& $\begin{array}{l}\text { Educación, Q-Sanitarias, R-Artistas y S-Otros. } \\
\text { Relación entre los activos disponibles líquidos no inmovilizados en el proceso } \\
\text { de explotación y aquellos que precisan solo de la fase de cobro para }\end{array}$ \\
Endeudamiento & $\begin{array}{l}\text { transformarse en tesorería, con los recursos ajenos con vencimiento a un año. } \\
\text { Porcentaje que representan los pasivos corrientes y no corrientes sobre el total } \\
\text { de la estructura financiera. }\end{array}$ \\
Rentabilidad económica & Beneficios antes de intereses, impuestos y depreciación (EBITDA) sobre el \\
total de activo.
\end{tabular}

Fuente: Elaboración propia.

\subsection{Metodología}

En los últimos años se han realizado importantes avances en el desarrollo de distintas metodologías para estimar y analizar los modelos de variables dependientes limitada en un contexto espacial. En este grupo de modelos existe una amplia variedad de especificaciones en función de las características de la variable analizada. Dada su extendida aplicabilidad en los procesos económicos, han sido los modelos probit espaciales, en los que la variable latente presenta estructura de dependencia espacial, los más estudiados. Distintas estrategias de estimación han sido propuestas para la estimación de estos modelos. Así, los modelos espaciales probit se han estimado por máxima verosimilitud (McMillen, 1992; Murdoch, et al., 2003), mínimos cuadrados ponderados (McMillen, 1992) y mediante estimadores obtenidos a partir del método generalizado de los momentos (Pinkse y Slade, 1998). Estos métodos de estimación suponen el desarrollo de procesos complicados en los que se requiere un importante coste temporal y que pueden llevar problemas de estimación en muestras pequeñas (LeSage y Pace, 2009). Es por ello, que el proceso de estimación Bayesiano (MCMC) está teniendo una importante relevancia últimamente (Wilhem y de Matos, 2013). Este proceso de estimación es más sencillo en su cómputo y por ello se ha utilizado esta metodología para obtener la estimación de los coeficientes del modelo probit espacial que proponemos en este trabajo.

El modelo espacial probit es representado en la siguiente expresión :

$$
\begin{gathered}
\mathrm{y}=\left\{\begin{array}{l}
1 \text { si } y^{*} \geq 0 \\
0 \text { si } y^{*}<0
\end{array}\right. \\
\mathrm{y}^{*}=\rho \mathrm{Wy}{ }^{*}+\mathrm{X} \beta+\varepsilon, \quad \varepsilon \sim \operatorname{MVN}\left(0, \sigma^{2} \mathrm{I}_{\mathrm{n}}\right) .
\end{gathered}
$$


donde $\mathrm{Wy}^{*}$ es una vector columna de dimensión $\mathrm{n}$ correspondiente al retardo espacial de la variable dependiente inobservada $\mathrm{y}^{*}$, $\mathrm{W}$ la matriz de pesos espaciales de dimensión nxn, $\rho$ es el parámetro autorregresivo espacial, y es el valor observado de la variable dependiente limitada, $\mathrm{X}$ es una matriz nxk de variables explicativas, $\beta$ es el vector de $k$ parámetros, $\mathrm{I}_{\mathrm{n}}$ es una matriz identidad de dimensión nxn. En el caso de $\rho=0$, el modelo espacial probit será un modelo binario probit estándar. Para llevar a cabo el proceso de estimación del modelo espacial (2) relajamos el supuesto de interdependencia estricta usado en los modelos probit tradicionales (Franzese y Hays, 2009). De este modo, permitimos que cambios en una variable explicativa de una empresa impacten en el valor de sus empresas vecinas, definidas a partir de la matriz de contactos W. En nuestra aplicación empírica, el modelo indicaría que un incremento en un factor que afecta de forma positiva a la probabilidad de fracaso de una empresa no solo aumentaría la probabilidad de bancarrota de dicha empresa sino también de las empresas que hemos considerado como empresas vecinas. Por tanto, se identifica un efecto marginal cuya cuantificación necesitaría de un análisis detallado de los coeficientes estimados. LeSage y Pace (2009) denominan a estos efectos marginales como directos, indirectos y totales. En el contexto del modelo probit espacial, estos impactos son cuantificados a partir de la siguiente expresión (3):

$$
\frac{\partial E\left[y \mid x_{r}\right]}{\partial x_{r}}=\varphi\left(S^{-1} I_{n} \bar{x}_{r} \beta_{r}\right) \otimes S^{-1} I_{n} \beta_{r}
$$

donde $x_{r}$ es la variable explicativa $r, \bar{x}_{r}$ es su valor medio, $\beta_{r}$ es el coeficiente probit estimado, $\varphi($.$) es la función de densidad normal. S^{-1}=\left(I_{n}-\rho W\right)^{-1}$ y $I_{n}$ es una matriz identidad de dimensión $n x n$. Por tanto, el valor esperado de la variable dependiente resultante de un cambio en la variable explicativa $x_{r}$ es el resultado del producto de dos matrices. El impacto directo del cambio en $x_{r}$ está representado por los elementos de la diagonal principal de la matriz resultante de la (3), y el impacto total del cambio en $x_{r}$ se obtiene como el valor medio de la suma de la fila de (3) correspondiente a $r$. El impacto indirecto se obtiene como diferencia entre el impacto total y el impacto directo.

\subsubsection{La matriz de pesos espaciales}

La modelización probit espacial requiere la utilización de una matriz de ponderaciones W. Esta matriz captura las relaciones de vecindad entre las distintas unidades de análisis como suele ser habitual en econometría espacial.

$$
W=\left[\begin{array}{cccc}
0 & w_{12} & \cdots & w_{1 n} \\
w_{21} & 0 & \cdots & w_{2 n} \\
\cdots & \cdots & \cdots & \cdots \\
w_{n 1} & w_{n 2} & \cdots & 0
\end{array}\right]
$$


donde cada término $\mathrm{w}_{\mathrm{ij}}(\mathrm{i}, \mathrm{j}=1, \ldots, \mathrm{n})$ es igual a uno si las empresas $i$ y $j$ son consideradas vecinas y cero en otro caso. Por definición, los elementos de la diagonal principal son iguales a 0 , lo que significa que ningún elemento es su propio vecino. En nuestro caso, basamos este análisis en una matriz de pesos basada en el criterio de vecindad de los k-vecinos más próximos. Por ejemplo, si consideramos las tres empresas más próximas a cada empresa analizada $(\mathrm{k}=3)$ estaremos suponiendo un esquema de conexión como muestra la Figura 1 (Panel A), mientras que si consideramos un criterio de vecindad basado en los cinco vecinos más próximos $(\mathrm{k}=5)$ tendremos un esquema de conexión como el mostrado en la Figura 1 (Panel B).

Figura 1

Ejemplo k-vecinos más cercanos

Panel A ( $k=3)$

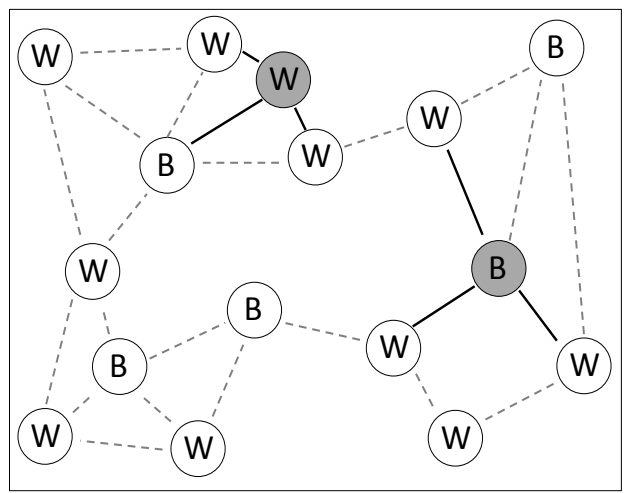

Panel B ( $k=5)$

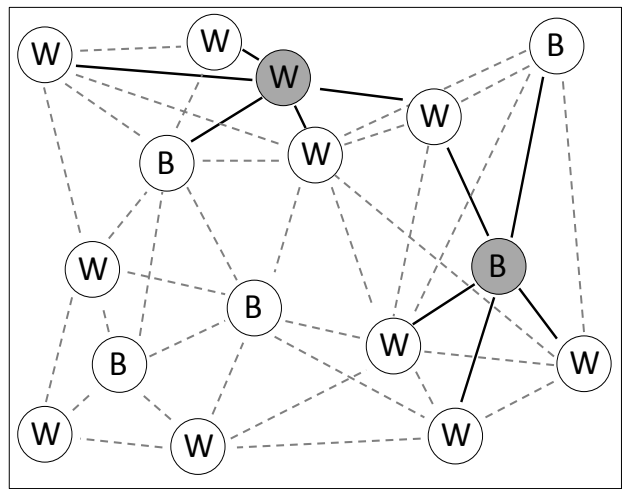

Fuente: Elaboración propia.

\section{RESULTADOS}

\subsection{Análisis descriptivo}

La muestra se divide en dos grupos de empresas, las que están en situación de fracaso y las que no están. Del total de las 2.710 empresas que componen la muestra, 448 presentan situación de fracaso, lo que supone una tasa de fracaso en torno al $17 \%$.

La Tabla 2 muestra la situación de fracaso empresarial atendiendo al criterio de definición económica utilizado en este trabajo, al subsector de actividad económica, al tamaño y edad. Respecto a la composición empresarial, un $37,79 \%$ de las empresas pertenecen al subsector de actividades comerciales y un porcentaje inferior $(17,71 \%)$ se dedican a actividades profesionales, científicas y técnicas. Las empresas inmobiliarias, con un porcentaje del 11,70\% se sitúan en tercer lugar. El resto de los sectores presentan porcentajes menores. Respecto 
a la situación de fracaso empresarial, son los subsectores de hostelería, actividades inmobiliarias y actividades científicas y técnicas, los que presentan un porcentaje más elevado de empresas fracasadas. En estos casos, el porcentaje de empresas fracasadas $(11,38 \%, 14,51 \%$ y $18,30 \%$ respectivamente) supera el porcentaje de empresas no fracasadas en dichos subsectores $(6,10 \%, 11,14 \%$ y $17,60 \%$ respectivamente). Además, superan el porcentaje de fracaso sobre el total empresarial. Este resultado es de esperar dado que el periodo de análisis considerado 2011-2013 corresponde al periodo de crisis financiera internacional con una alta repercusión sobre el sector inmobiliario y al carácter procíclico de los subsectores de hostelería y servicios de profesional independientes.

\section{Tabla 1}

Distribución de empresas por sub-actividades del sector servicios, tamaño y edad. Media 2011-2013

\begin{tabular}{|c|c|c|c|c|c|c|}
\hline & \multicolumn{2}{|c|}{ Fracaso } & \multicolumn{2}{|c|}{ No Fracaso } & \multicolumn{2}{|c|}{ Total Empresas } \\
\hline & $\begin{array}{c}\mathrm{N}^{\circ} \\
\text { Empresas }\end{array}$ & $\begin{array}{l}\% \text { sobre total } \\
\text { de empresas } \\
\text { fracasadas }\end{array}$ & $\begin{array}{c}\mathrm{N}^{\circ} \\
\text { Empresas }\end{array}$ & $\begin{array}{c}\text { \% sobre total } \\
\text { de empresas } \\
\text { no } \\
\text { fracasadas }\end{array}$ & $\begin{array}{c}\mathrm{N}^{\circ} \\
\text { Empresas }\end{array}$ & $\begin{array}{c}\% \text { sobre } \\
\text { el total }\end{array}$ \\
\hline \multicolumn{7}{|c|}{ Subsector de Actividad } \\
\hline G. Comercio & 123 & $27,46 \%$ & 901 & $39,83 \%$ & 1.024 & $37,79 \%$ \\
\hline H. Transporte & 23 & $5,13 \%$ & 126 & $5,57 \%$ & 149 & $5,50 \%$ \\
\hline I. Hostelería & 51 & $11,38 \%$ & 138 & $6,10 \%$ & 189 & $6,97 \%$ \\
\hline J. Información & 19 & $4,24 \%$ & 86 & $3,80 \%$ & 105 & $3,87 \%$ \\
\hline L. Inmobiliarias & 65 & $14,51 \%$ & 252 & $11,14 \%$ & 317 & $11,70 \%$ \\
\hline M. Científicas y técnicas & 82 & $18,30 \%$ & 398 & $17,60 \%$ & 480 & $17,71 \%$ \\
\hline N. Administración & 34 & $7,59 \%$ & 123 & $5,44 \%$ & 157 & $5,79 \%$ \\
\hline O. Defensa & 1 & $0,22 \%$ & 4 & $0,18 \%$ & 5 & $0,18 \%$ \\
\hline P. Educación & 5 & $1,12 \%$ & 45 & $1,99 \%$ & 50 & $1,85 \%$ \\
\hline Q. Sanitarias & 11 & $2,46 \%$ & 87 & $3,85 \%$ & 98 & $3,62 \%$ \\
\hline R. Artistas & 17 & $3,79 \%$ & 54 & $2,39 \%$ & 71 & $2,62 \%$ \\
\hline S. Otros & 17 & $3,79 \%$ & 48 & $2,12 \%$ & 65 & $2,40 \%$ \\
\hline \multicolumn{7}{|c|}{ Tamaño empresarial por número de trabajadores } \\
\hline Micro & 405 & $90,40 \%$ & 1.805 & $79,80 \%$ & 2.210 & $81,55 \%$ \\
\hline Pequeña & 36 & $8,04 \%$ & 401 & $17,73 \%$ & 437 & $16,13 \%$ \\
\hline Mediana & 7 & $1,56 \%$ & 56 & $2,48 \%$ & 63 & $2,32 \%$ \\
\hline \multicolumn{7}{|c|}{ Edad de la empresa } \\
\hline Joven (<15 años) & 301 & $67,19 \%$ & 1311 & $57,96 \%$ & 1.612 & $59,48 \%$ \\
\hline Madura ( $\geq 15$ años) & 147 & $32,81 \%$ & 951 & $42,04 \%$ & 1.098 & $40,52 \%$ \\
\hline Total & 448 & $16,53 \%^{(+)}$ & 2262 & $83,47 \%^{(t)}$ & 2.710 & - \\
\hline
\end{tabular}

${ }^{(+)}$porcentaje sobre el total de empresas.

Fuente: Elaboración propia.

Para analizar la relación existente entre el tamaño empresarial y su situación de fracaso categorizamos la variable tamaño empresarial siguiendo la recomendación de la Comisión Europea 6 de mayo de 2003. De este modo, la microempresa es aquella con menos de diez empleados, la pequeña empresa aquella que 
tiene entre diez y cincuenta trabajadores y la empresa mediana aquella que tiene más de cincuenta y menos de doscientos cincuenta. La Tabla 2 muestra estos resultados. De las empresas de la muestra, las identificadas como microempresas supusieron el 81,55\% (2.210 empresas), seguido por las pequeñas empresas con 16,13\% (437 empresas) y en menor medida, las empresas medianas con casi un 2,32\% (63 empresas). Esta distribución corresponde con la distribución poblacional de empresas por tamaños (DIRCE, 2013). Por otro lado, si se consideran exclusivamente las que están en situación de fracaso, las microempresas ocupan el primer lugar con un 90,40\% (405 empresas), en segundo lugar, las pequeñas empresas con 8,04\% (36 empresas) y, en un número muy reducido, las medianas con 1,56\%. Estos porcentajes confirman resultados de estudios anteriores apuntando acerca de una relación negativa entre el tamaño y la probabilidad de fracaso empresarial (Calvo-Flores, 2007; Back, 2005; Watson y Everett, 1996).

Por último, atendiendo a la edad de la empresa se ha categorizado la variable edad considerando como empresas jóvenes aquellas con menos de quince años desde su constitución y empresas maduras aquellas que tienen más de quince años $^{3}$. Los resultados de la incidencia del factor edad en el fracaso empresarial se muestran en las últimas filas de la Tabla 2 . Un 59,48\% de las empresas (1.612 empresas) son jóvenes, el resto son maduras 42,04\% (1.098 empresas) identificando una tasa de fracaso en empresas jóvenes de un 67,19\% (301 empresas), mientras que en las empresas maduras es del 32,81\% (147 empresas). Estos resultados confirman lo demostrado por trabajos previos (Misas, 2008) respecto a la mayor probabilidad de fracaso de las empresas jóvenes frente a las maduras.

\subsection{Distribución espacial}

La Figura 2 muestra la localización de las empresas, donde los puntos grises identifican empresas sanas y los negros empresas fracasadas. En esta figura se aprecia una tendencia de aglomeración hacia la zona centro norte (centro del municipio de Murcia). Si nos centramos exclusivamente en empresas fracasadas, se observa que el comportamiento es similar a las no fracasadas, éstas tienden a concentrarse en el núcleo urbano de la ciudad y tienden a estar rodeadas de empresas también fracasadas. Por tanto, esta primera aproximación parece indicar acerca de un efecto positivo en el fracaso motivado por la distancia entre

\footnotetext{
${ }^{3}$ Estudios previos consideran más valores de referencia para categorizar la edad empresarial. Así, Bergel y Udell (1998) consideran como empresas embrión aquellas que tienen menos de dos años, adolescentes de tres a cuatro años, empresas jóvenes edad de cinco a quince años y empresas maduras más de quince años. En nuestro caso al realizar esta clasificación nos quedamos con prácticamente ninguna empresa en las por debajo de los quince años y, por tanto, decidimos seleccionar el criterio de los quince años como punto de corte.
} 
empresas. Es decir, empresas fracasadas tienden a estar rodeadas de empresas fracasadas.

Figura 2

Distribución geográfica de las empresas

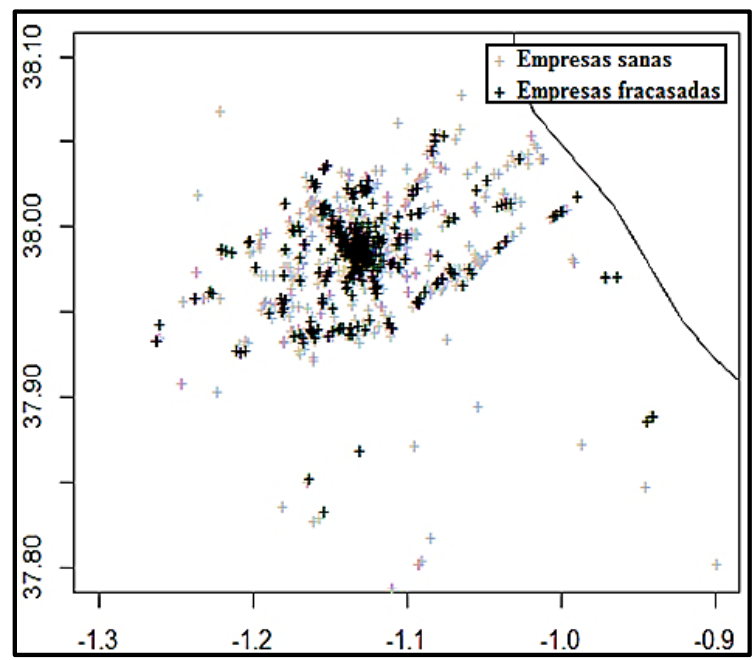

Fuente: Elaboración propia utilizando software libre R.

Con el propósito de contrastar el contagio en el fracaso empresarial en el grupo de empresas localizadas geográficamente, en la siguiente sección se propone la estimación de un modelo probit espacial.

\subsection{Modelo de regresión espacial probit}

Para la estimación del modelo espacial probit se ha utilizado la matriz de pesos basada en los quince vecinos más cercanos $(\mathrm{k}=15)$. La selección de este grado de vecindad se deriva de un proceso de estimación iterativo en el que se seleccionó aquel modelo cuyo coeficiente de autocorrelación espacial $(\rho)$ presentaba una mayor significatividad. La variable dependiente de dicho modelo hace referencia al fracaso empresarial tomando como referencia el periodo 2011-2013 mientras que los ratios financieros utilizados como factores explicativos se corresponden al año 2010. Finalmente, los subsectores incluidos en esta estimación son aquellos que suponen más de un 5\% sobre la composición muestral.

La Tabla 3 muestra los resultados de los coeficientes estimados de los modelos probit y probit espacial. Los signos de las distintas variables coinciden con los resultados obtenidos en la literatura anterior. El test de cocientes de verosimilitud $(\mathrm{LR})$ es significativo ( $\mathrm{p}$-valor $<0,05)$ indicando un mejor ajuste del 
modelo probit espacial frente al modelo probit. Este es el hecho más destacable para nuestra investigación ya que confirma la presencia de un coeficiente autorregresivo $(\rho)$ significativo concluyendo a favor de la existencia de una estructura de autocorrelación espacial positiva en el fracaso empresarial.

Tabla 3

Resultados de la estimación del modelo probit de fracaso empresarial. Año 2013

\begin{tabular}{|c|c|c|c|c|}
\hline & \multicolumn{2}{|c|}{ Probit } & \multicolumn{2}{|c|}{ Probit espacial } \\
\hline & Beta & (p-valor) & Beta & (p-valor) \\
\hline Liquidez & $-0,0019^{* * \star}$ & $(0,000)$ & $-0,0020^{* \star \star}$ & $(0,000)$ \\
\hline Rentabilidad & $-0,0227^{\star \star \star}$ & $(0,000)$ & $-0,0231^{\star \star *}$ & $(0,000)$ \\
\hline Endeudamiento & $0,0147^{\star \star \star}$ & $(0,000)$ & $0,0143^{\star \star \star}$ & $(0,000)$ \\
\hline Tamaño & $-0,1643^{* \star *}$ & $(0,000)$ & $-0,1711^{\star \star *}$ & $(0,000)$ \\
\hline Edad & $-0,1521^{\star *}$ & $(0,013)$ & $-0,2188^{\star \star *}$ & $(0,000)$ \\
\hline Comercio & 0,0535 & $(0,587)$ & 0,0317 & $(0,734)$ \\
\hline Transporte & $-0,1036$ & $(0,564)$ & $-0,1498$ & $(0,401)$ \\
\hline Hostelería & $0,1584^{*}$ & $(0,087)$ & $0,1179^{*}$ & $(0,073)$ \\
\hline Información & 0,0308 & $(0,896)$ & $-0,0300$ & $(0,891)$ \\
\hline Inmobiliarias & $0,1293^{* \star \star}$ & $(0,005)$ & $0,1449^{\star \star \star}$ & $(0,003)$ \\
\hline Científicas y técnicas & $0,2526^{\star}$ & $(0,064)$ & $0,2956^{* *}$ & $(0,020)$ \\
\hline Efecto espacial $(\rho)$ & -- & -- & $0,1831^{* *}$ & $(0,035)$ \\
\hline LR (probit vs probit espacial) & & & $12,924^{* \star}$ & $(0,050)$ \\
\hline
\end{tabular}

La interpretación de los efectos marginales en este modelo probit espacial es más compleja y a la vez mas informativa que para un modelo probit no espacial. Estos efectos marginales requieren de un proceso de descomposición de los efectos totales en efectos directos e indirectos (LeSage y Pace, 2009). Como se indicó en la sección 3.3, los efectos directos tienen en cuenta el efecto derivado del cambio en una de las variables explicativas en una empresa determinada $i$ en la probabilidad de fracaso de dicha empresa $i$. Los efectos indirectos evalúan el efecto producido por un cambio en una de las variables explicativas de una de las empresas vecinas $j$ a la empresa $i$ sobre la probabilidad de fracaso de la empresa $i$.

La Tabla 4 muestra los resultados de los efectos marginales obtenidos del modelo probit espacial. Si consideramos las variables características de la empresa, edad y tamaño, los resultados reflejan una conducta ya descrita en la literatura. Las empresas más jóvenes (Misas, 2008; Shumway, 2001), así como las más pequeñas (Calvo-Flores, 2007; Back, 2005; Watson y Everett, 1996), tienen mayor riesgo de fracaso que las empresas maduras y/o de mayor tamaño. Nótese que las variables originales están expresadas en logaritmos y por tanto se refieren al cambio en probabilidad cuando el log x se incrementa en una unidad. Además, este mayor riesgo se mantiene en el caso de que las empresas vecinas 
sean empresas pequeñas y/o jóvenes. En cuanto al sector, el hecho de que una empresa pertenezca al subsector hostelería aumenta la probabilidad de fracaso en 1,3 puntos porcentuales, en 6,0 si es del sector inmobiliario y en un 4,8 en el caso de que sean actividades científicas y técnicas respecto al resto de los sectores no incluidos en esta clasificación que se toman como sector de referencia. Estos efectos positivos y significativos se mantienen el caso de que estas empresas analizadas estén rodeadas de empresas pertenecientes a dichos subsectores. Es decir, el hecho de que una empresa esté localizada próxima a empresas del sector de hostelería, inmobiliario o de actividades científicas y técnicas aumenta su probabilidad de fracaso. Por lo que respecta a las variables financieras, encontramos un efecto directo negativo y significativo en los ratios de liquidez y rentabilidad. Por lo tanto, el hecho de tener mayores niveles de liquidez y/o rentabilidad en la empresa analizada disminuye la probabilidad de fracaso de dicha empresa. El ratio de endeudamiento presenta un resultado contrario. Así, encontramos con un signo positivo y significativo por lo que empresas con mayores niveles de endeudamiento aumentan su probabilidad de fracaso. Estos efectos presentan coeficientes significativos más elevados para los efectos indirectos. Es decir, el hecho de que una empresa se encuentre rodeada de empresas con valores altos de rentabilidad y/o bajos de endeudamiento disminuye la probabilidad de fracaso de dicha empresa.

Tabla 4

Efectos totales, directos e indirectos del probit espacial. Año 2013

\begin{tabular}{lccc}
\hline & Directo & Indirecto & Total \\
\hline Liquidez & $-0,0002^{* *}$ & $-0,0000^{* *}$ & $-0,0002^{* *}$ \\
Rentabilidad & $-0,0030^{* *}$ & $-0,0002^{* *}$ & $-0,0032^{* *}$ \\
Endeudamiento & $0,0017^{* *}$ & $0,0003^{* *}$ & $0,0020^{* *}$ \\
Tamaño & $-0,0194^{* *}$ & $-0,0044^{* *}$ & $-0,0238^{* *}$ \\
Edad & $-0,0243^{* *}$ & $-0,0059^{* *}$ & $-0,0302^{* *}$ \\
Comercio & 0,0051 & $-0,0006$ & 0,0045 \\
Transporte & $-0,0245$ & 0,0038 & $-0,0207$ \\
Hostelería & $0,0138^{* *}$ & $0,0027^{* *}$ & $0,0165^{* *}$ \\
Información & $-0,0049$ & 0,0010 & $-0,0039$ \\
Inmobiliarias & $0,0609^{* *}$ & $0,0016^{* *}$ & $0,0625^{* *}$ \\
Científicas y técnicas & $0,0484^{* *}$ & $0,0073^{* *}$ & $0,0411^{* *}$ \\
\hline
\end{tabular}

${ }^{* *}$ Significativo al 1\%; ${ }^{* *}$ significativo al 5\% ; ${ }^{*}$ significativo al 10\%.

Fuente: Elaboración propia utilizando software libre R.

\section{CONCLUSIONES}

Este trabajo tiene por objetivo contrastar el efecto de contagio en el fracaso empresarial como consecuencia de la proximidad geográfica entre empresas. Para ello, se ha considerado una muestra de pymes del sector servicios localizadas en el municipio de Murcia y mediante la aplicación de un modelo de regresión probit espacial se ha cuantificado la probabilidad de fracaso en una 
empresa como consecuencia de los resultados financieros y atributos de empresas próximas. Los resultados confirman la existencia de un efecto del contagio en el fracaso empresarial. Además, de los resultados obtenidos se deduce que la probabilidad de fracaso empresarial aumenta (disminuye) si las empresas próximas aumentan su endeudamiento (rentabilidad). También muestran que la probabilidad de fracaso será mayor si las empresas vecinas pertenecen al subsector inmobiliario, de hostelería o de actividades dedicadas a servicios de profesionales independientes. Por último, si la empresa en cuestión está rodeada de empresas de reducido tamaño o empresas jóvenes, su probabilidad de fracaso también aumenta.

En síntesis, de la estimación del modelo probit espacial obtenemos que la probabilidad de fracaso de una empresa no solo depende de los factores internos de la empresa, sino también de características de empresas geográficamente próximas. Este trabajo evidencia la necesidad de considerar la interacción espacial entre observaciones como otro elemento más en el análisis de los determinantes del fracaso empresarial.

Por último, somos conscientes de este trabajo tiene al menos dos limitaciones que nos gustaría resaltar. En primer lugar es posible que el periodo analizado, correspondiente con una profunda crisis económica, tenga impacto sobre los resultados. Sería necesario aplicar esta metodología a una muestra con mayor dimensión temporal y que no abarque un periodo de crisis económica tan profunda que ha podido contaminar parcialmente los resultados obtenidos. En segundo lugar, en este trabajo se han considerado la matriz $\mathrm{W}$ basada en los quince vecinos más próximos. Este criterio, aunque en este caso ofrece un coeficiente autorregresivo con mayor significatividad, es ciertamente subjetivo y sería necesario también explorar la significatividad del efecto espacial con otras muestras que permitan comparar resultados.

\section{REFERENCIAS BIBLIOGRÁFICAS}

ADDOUM, J.; KUMAR, A. y LE, N. (2014). "Contagious Negative Sentiment and Corporate Policies: Evidence from Local Bankruptcy Filings". Finance Meeting EUROFIDAI-AFFI Paper.

ALTMAN, E. (1968). "Financial ratios, discriminant analysis and the prediction of corporate bankruptcy". The Journal of Finance, 23(4), 589-609.

AUDRETSCH, D. y FELDMAN, M. (1996). "R\&D spillovers and the geography of innovation and production". The American Economic Review, 630-640.

BACK, P. (2005). "Explaining financial difficulties based on previous payment behavior, management background variables and financial ratios". European Accounting Review, 14(4), 839-868. 
BATTISTON, S.; GATTI, D.; GALLEGATI, M.; GREENWALD, B. y STIGLITZ, J. (2007). "Credit chains and bankruptcy propagation in production networks". Journal of Economic Dynamics and Control, 31(6), 2061-2084.

BEAVER, W. (1966). "Financial ratios as predictors of failure". Journal of Accounting Research, 71-111.

BERGER, A. N. Y UDELL, G. F. (1998). "The economics of small business finance: The roles of private equity and debt markets in the financial growth cycle". Journal of Banking \& Finance 22 (6-8): 613-673.

CALVO-FLORES, A.; GARCÍA, D. y GUIJARRO, A. (2006). "Tamaño, antigüedad y fracaso empresarial". Working paper $n^{\circ} 1$. Grupo Interuniversitario de Investigación. Análisis Estratégico para el Desarrollo de la Pyme.

CINCA, C.; MOLINERO, C. y LARRAZ, J. (2005). "Country and size effects in financial ratios: A European perspective". Global Finance Journal, 16(1), 26-47.

CONTABILIDAD REGIONAL DE ESPAÑA (CRE). Base 2010, Serie 2010-2014, del Instituto Nacional de Estadística (INE). http://www.ine.es/daco/daco42/cre00/b2010/ dacocre_base2010.htm [Último acceso: septiembre de 2015].

DEAKIN, E. (1972). "A discriminant analysis of predictors of business failure". Journal of accounting Research, 167-179.

DEGRYSE, H. y ONGENA, S. (2005). "Distance, lending relationships, and competition". The Journal of Finance, 60(1), 231-266.

DITTMAR, A. y DUCHIN, R. (2014). "Looking in the rear view mirror: the effect of managers' professional experience on corporate financial policy". Ross School of Business Paper, (1221).

EDMISTER, R. (1972). "An empirical test of financial ratio analysis for small business failure prediction". Journal of Financial and Quantitative analysis, 7(2), 1477-1493.

FERNÁNDEZ, M. y GUTIERREZ, F. (2012). "Variables y modelos para la identificación y predicción del fracaso empresarial: revisión de la investigación empírica reciente". Revista de Contabilidad, 15(1).

FRANZESE JR, R. y HAYS, J. (2009). "Empirical modeling of spatial interdependence in time-series cross-sections". Methoden der vergleichenden Politik-und Sozialwissenschaft (pp. 233-261). VS Verlag für Sozialwissenschaften.

GATTI, D.; DI GUILMI, C.; GAFFEO, E.; GIULIONI, G.; GALLEGATI, M. y PALESTRINI, A (2005). "A new approach to business fluctuations: heterogeneous interacting agents, scaling laws and financial fragility". Journal of Economic Behavior and Organization, 56(4), 489-512.

GEPP, A. y KUMAR, K. (2012). "Business failure prediction using statistical techniques: A review". Some Recent Developments in Statistical Theory and Applications, 1-25.

GRANOVETTER, M. (1985). "Economic action and social structure: the problem of embeddedness". American Journal of Sociology, 481-510.

HAUNSCHILD, P. y BECKMAN, C. (1998). "When Do Interlocks Matter?: Alternate Sources of Information and Interlock Influence". Administrative Science Quarterly, 815-844.

HERTZEL, M. y OFFICER, M. (2012). "Industry contagion in loan spreads". Journal of Financial Economics, 103(3), 493-506.

HERTZEL, M.; LI, Z.; OFFICER, M. y RODGERS, K. (2008). "Inter-firm linkages and the wealth effects of financial distress along the supply chain". Journal of Financial Economics, 87(2), 374-387. 
KOLAY, M.; LEMMON, M. y TASHJIAN, E. (2015). Spreading the Misery? Sources of Bankruptcy Spillover in the Supply Chain. San Diego Meetings Paper.

LANG, L. y STULZ, R. (1992). "Contagion and competitive intra-industry effects of bankruptcy announcements". Journal of Financial Economics, 32(1), 45-60.

LE, N. (2012). Spillover effects of intra-industry bankruptcy filings on firms' cash holding policy. Northern Finance Association Meeting.

LESAGE, J. y PACE, R. (2009). Introduction to Spatial Econometrics. Boca Raton, FL: CRC Press.

LI, H. y SUN, J. (2011). "Principal component case-based reasoning ensemble for business failure Prediction". Information and Management, 48(6), 220-227.

LI, H. y XIONG, T. (2012). Predicting business risk using combined case-based reasoning in Euclidean space. World Automation Congress (WAC), 1-6.

MATÉ, M.; HERNÁNDEZ, G.; SÁNCHEZ, J. y MÍNGUEZ, A. (2013). "Are there spill-over effects into financial behaviour of SMEs?". Trimestre Económico, 80(320), 841-867.

MATÉ, M.; GARCÍA D. y LÓPEZ F. (2009). "Spatial effects in the productivity convergence of Spanish industrial SME's". Spanish Journal of Finance and Accounting, 38(141), 13-36.

MATEOS, A. y LÓPEZ A. (2011). "Developing a business failure prediction model for cooperatives: Results of an empirical study in Spain". African Journal of Business Management, 5(26), 10565-10576.

MCCULLOCH, W. y PITTS, W. (1943). "A logical calculus of the ideas immanent in nervous activity". The bulletin of mathematical biophysics, 5(4), 115-133.

MCMILLEN, D. (1992). "Probit with spatial autocorrelation". Journal of Regional Science, 32(3), 335-348.

MISAS, M. (2008). "Análisis del fracaso empresarial en Andalucía. Especial referencia a la edad de la empresa". Cuadernos de Ciencias Económicas y Empresariales, 1(54), 35-56.

MURDOCH, J.; SANDLER, T. y VIJVERBERG, W. (2003). "The participation decision versus the level of participation in an environmental treaty: A spatial probit analysis". Journal of Public Economics, 87 (2), 337-362.

OHLSON, J. (1980). "Financial ratios and the probabilistic prediction of bankruptcy". Journal of Accounting Research, 109-131.

PARSONS, A.; SULAEMAN, J. y TITMAN, S. (2014). "The geography of financial misconduct". (No. w20347) National Bureau of Economic Research.

PINKSE, J. y SLADE, M. (1998). "Contracting in space: An application of spatial statistics to discrete-choice models". Journal of Econometrics, 85(1), 125-154.

PIRINSKY, C. y WANG, Q. (2010). Geographic location and corporate finance: A review. Handbook of Emerging Issues in Corporate Governance. London, UK, World Scientific Publishing.

QUINTANA, M.; GALLEGO, A. y PASCUAL, M. (2012). "Análisis del fracaso empresarial por sectores: factores diferenciadores". Pecunia: Revista de la Facultad de Ciencias Económicas y Empresariales, (1), 53-83.

RODRÍGuEZ, M.; PIÑEIRO, C. y DE LLANO, P. (2015). Predicción de insolvencia y fracaso financiero: medio siglo después de Beaver (1966). Avances y nuevos resultados. XVIII Congreso AECA, Cartagena, Octubre 2015. 
ROKACH, L. y MAIMON, O. (2008). Data Mining with Decision Trees: Theory and Applications. World Scientific Publishing.

SHUMWAY, T. (2001). "Forecasting bankruptcy more accurately: A simple hazard model". The Journal of Business, 74(1), 101-124.

TURETSKY, H. y MCEWEN, R. (2001). "An Empirical Investigation of Firm Longevity: A Model of the Ex Ante Predictors of Financial Distress". Review of Quantitative Finance and Accounting, 16(4). 323-343.

WATSON, J. y EVERETT, J. (1996). "Small Business Failure Rates: Choice of Definition and the Size Effect". The Journal of Entrepreneurial and Small Business Finance, 5(3), 271-285.

WILHELM, S. y DE MATOS, M. (2013). "Estimating spatial probit models in R". The $R$ Journal, 5(1), 130-143.

XU, W.; XIAO, Z.; DANG, X.; YANG, D. y YANG, X. (2014). "Financial ratio selection for business failure prediction using soft set theory". Knowledge-Based Systems, 63, 5967.

YOUN, H. y GU, Z. (2010). "Predicting Korean lodging firm failures: An artificial neural network model along with a logistic regression model". International Journal of Hospitality Management, 29(1), 120-127. 